\title{
Implement Wireless Transceiver System Based On Convolutional Coding; Aided by Soft-Bit Decoding
}

\author{
Israa Hazem Ali \\ Communications Eng. Dept., Collage of Engineering, University of Diyala \\ pg_student75@yahoo.com
}

\begin{abstract}
The main aim with any modern digital communication systems is to provide error free data transmission. To achieve this aim, error control coding techniques are applied with these systems. In this paper, digital communication system was implemented based on convolutional code, aided by soft-bit decoding for error free transmission. Fixed length source encoder was represented with this work, and statistically described the output of the source encoder and save this description at the receiver as a priori knowledge about the source encode. These a priori knowledge were exploited at the receiver to improve the performance of the proposed system in term of BER by conceal the errors occurred through the transmission of information over AWGN channel, aided the convolutional encoder. Significant improvements were obtained with this proposed system.
\end{abstract}

Index Terms-Convolutional code, soft-bit decoding, BPSK, AWGN channel.

\section{INTRODUCTION}

Two objectives related to digital information transmission and storage systems; which are efficiency and reliability; have increasingly demanded in resent year by governments and even individuals for processing the data at high speed requirements in communication systems. The major requirement to design digital communication systems is combining of communication and computer technology to obtain reliable reproduction of data to avoid concern of designer from control of errors. The researcher Shannon approved in 4th decade from 20th century in work paper that can be protected the rate of information transmission or storage and reduces the errors by noisy channel or storage medium [1]. Since then, the work has been constantly to solve the problem of errors produced from the development of coding and decoding methods in a noisy environment. Today High-speed digital systems have contributed to the reliability required in modern communications systems and digital computers [2].

Error detection and correction coding is the technique by which errors that may introduced into transmitted digital signal as a consequence of transmission over a communication channel can be detected and corrected based to received signal. Error detection and error correction techniques are collectively called error control coding. Big difference obtained between the dysfunctional and operating communication systems by applying error control coding. Error control coding makes a significant revolution in telecommunications, like Internet, digital recording, and space inspection [3].

Forward error correction implies linear codes such as block and Convolutional codes. More explicitly, convolutional codes are linear sequences codes which have a generator matrix with additional structure, this property make the encoding process as a convolutional operation or filtering process. With a big demand for reliability, the convolutional codes become a popular techniques used in practical digital communication. The encoder with convolutional codes technique can be represented as series of 
digital filter with properties of linearity and time-invariant system, with additional effects, interleaving, applied to the output of filter. Comparable with the complexity of block encoder and decoder, the convolutional codes provide a significant performance, wherefore the Convolutional coding techniques are preferred in practice above block codes. Additionally, with convolutional codes the influential algorithm for soft-decision decoding was developed by Viterbi [2], [4].

With the block codes which are sub class of FEC, $\mathrm{k}$ bits are extracted from the transmitted information bits to produce $\mathrm{n}$ bits via channel encoder by adding $\mathrm{r}$ bits to the transmitted bits, contrary to convolutional codes which adds the redundancy to the input sequence of transmitted information, i.e. the transmitted bits are moved to the convolutional encoder as stream bits, operate on continuous streams of bits not partitioned into discrete blocks. On the other hand the coding rate still $=k / n$, the encoder accepting $\mathrm{k}$ bits at each time step then producing $\mathrm{n}$ bits for that step [5].

The performance of convolutional code was investigated with coding rate $2 / 3$ by [1], besides they used Viterbi algorithm for soft-decision decoding to evaluate the convolutional code performance, and the results show comparison between uncoded bit error rates with convolutional coded BER [4]. Different schemes were proposed for channel decoding depending on soft-decision decoding by [4-4] and [4-5] to improve their system's performance. Log-likelihood ratios (LLRs) were applied for softdecision decoding with [4-6, 4-7, 4-8]. Then soft-decision speech and audio decoders are suggested such as G.726 ADPCM, A-law PCM and GSM Full rate speech coding, high-quality PCM audio, and AMR-WB which used fixed length code words for the quantized source codes [5].

In this paper, the system of digital communication based on convolutional coding as a technique of error control coding has been implemented using MATLAB software, aided by soft bit decoding. The proposed system, which is shown in figure (1), consists of source of information block which selected as uniform distribution of random variable, these random variable are fed up to source encoder which represent a fixed length source coding process, the output of the source encoder is described statistically and moved to the channel encoder at the same time. The statistical description is exploited at the receiver to improve the performance of the proposed system in term of BER by conceal the errors occurred through the transmission of information over AWGN channel. The simplest form of modulation (BPSK) is used to modulate and prepare the data for transmission. The results show a significant improvement in terms of BER [6].

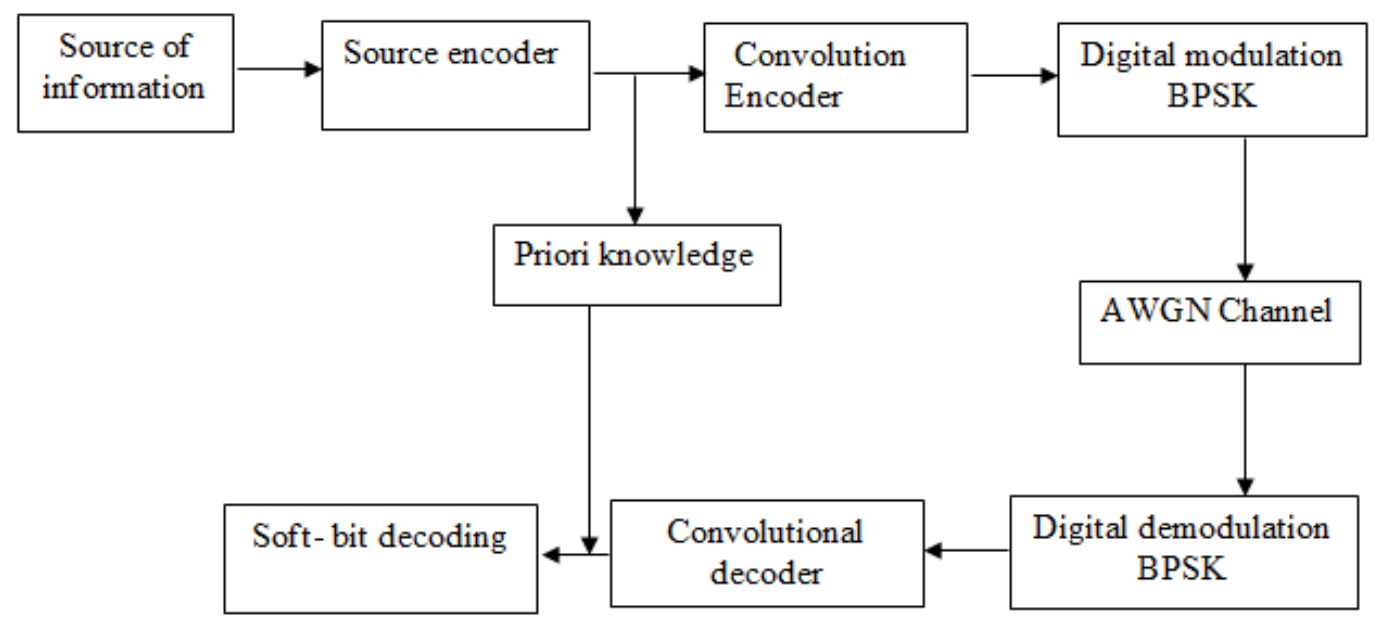

Fig. 1. Block Diagram of Proposed Digital Communication System 


\section{TRANSMITTER}

The complete proposed transmission system is shown in figure (1). The input discrete signal consists of series of samples varies among the minimum and maximum values, these samples are moved to scalar quantizer. The scalar quartier's output is a sequence of number of levels named $\mathrm{x}, \mathrm{x}=$ $\left[\mathrm{x}_{0}, \mathrm{x}_{1}, \mathrm{x}_{2}, \ldots \mathrm{x}_{\mathrm{i}}\right], \mathrm{x}_{\mathrm{i}} \in \mathrm{Qt}$ with $\mathrm{i}=\left[0,1,2, \ldots, 2^{\mathrm{B}}-1\right]$. Fixed length source encoder used to encode the output levels of quantizer with B bits resolution for each level, in this work the B is selected to be 5 bits.

The self-probability defined as the frequency of the symbol appearing at the output of the source encoder, which is statistically calculated. The vector which represents the self-probabilities $\mathrm{p}\left(\mathrm{x}_{\mathrm{i}}\right)$ has been saved in the decoder at the receiver as a priori knowledge describing the behavior of the source encoder output, in this work the order of self- probability selected as zero order priori knowledge [7].

The output of the source encoder are moved to the channel encoder after calculating the priori knowledge to these data, to carry out the process of adding redundancy to the transmitted sequence of bits. Convolutional encoder was used in this work to perform the channel coding function, due its capability of providing good performance and low cost implementation. A continuous sequence of transmitted information bits that using convolutional codes is mapped into continuous sequence of bits at encoder output, the decoding complexity depends on mapping process. With convolutional encoder the encoded bits output are memory output depended. Convolutional codes have a coding gain larger than block codes [8].

By using simple registers and adders one can achieve the convolutional encoder for convolutional codes. Convolutional codes defined by three parameters ( $\mathrm{n}, \mathrm{k}, \mathrm{L}$ ), when $\mathrm{n}$ represents the number of output bits according to $\mathrm{k}$ input bits to the encoder, and the third parameters $\mathrm{L}$ denotes the number of register stages (code memory depth). Besides the mentioned three parameters, there is an important parameter defined with convolutional codes, this parameter is constraint length and it is defined by the number of shift register plus the input. The efficiency of convolutional codes are measured by coding rate which represent the ratio of input bits $\mathrm{k}$ to the output bits $\mathrm{n}$, thus, $\mathrm{R}=\mathrm{k} / \mathrm{n}$ [9].

The procedures of convolutional encoder start with:

1- L elements of shift register memory, each element holding one bit.

2- The encoder consists of modulo-2 adders, and $n$ generator polynomials one for each adder.

Figure (2) illustrates the connection diagram of convolutional encoder used with this work, the encoder implemented with MATLAB and defined by these parameters:

1- $\quad \mathrm{L}=2$, number of shift register.

2- $\mathrm{K}=1$, one bit for each step time.

3- $\mathrm{N}=3$, three bit for each step time.

4- $\quad \mathrm{C}=3$, constraint length for the implemented convolutional encoder.

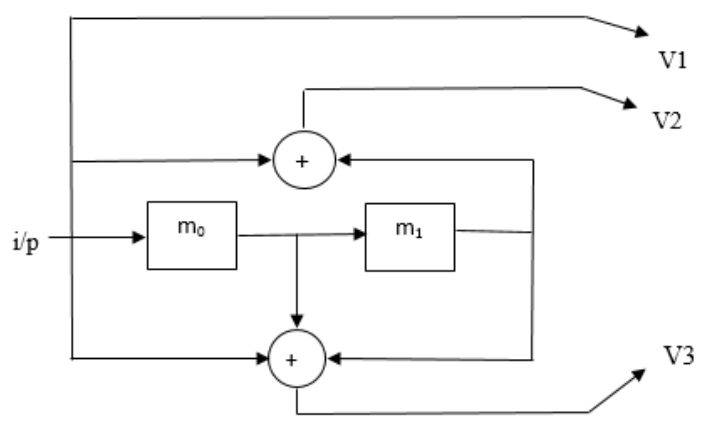

Fig. 2. CONNECTION Diagram OF CONVOLUTIONAL ENCODER 
The convolutional encoder can be representing by (connection diagram, state diagram, look up table, tree diagram, and trellis diagram), table one describes the look up table for the convolutional encoder used with this paper, the table shows all the probabilities of input bits and the corresponding output bit for each input, additionally it shows the state machine at each time step [3].

TABle 1. LOOK Up TABle For The CONVOlutional ENCODER

\begin{tabular}{|c|c|c|c|c|c|}
\hline Input data & Old state & Next state & \multicolumn{3}{|c|}{ Output } \\
\cline { 3 - 6 } & & & $\mathrm{V}_{1}$ & $\mathrm{~V}_{2}$ & $\mathrm{~V}_{3}$ \\
\hline 0 & 00 & 00 & 0 & 0 & 0 \\
\hline 0 & 10 & 01 & 0 & 0 & 1 \\
\hline 0 & 01 & 00 & 0 & 1 & 1 \\
\hline 0 & 11 & 01 & 0 & 1 & 0 \\
\hline 1 & 00 & 10 & 1 & 1 & 1 \\
\hline 1 & 10 & 11 & 1 & 1 & 0 \\
\hline 1 & 01 & 10 & 1 & 0 & 0 \\
\hline 1 & 11 & 11 & & & 1 \\
\hline
\end{tabular}

Figure (3) shows the state diagram for mention convolutional encoder, which can be exploited to implement the encoder as hardware device. The state diagram explains the state machine and all probabilities of output bits according to the input bits [3].

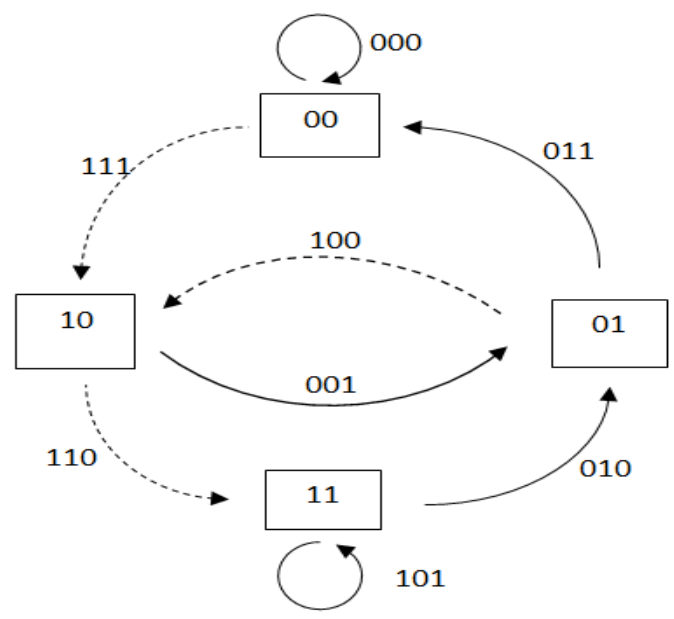

Fig. 3. State Diagram of Convolutional EnCODER

The sequence which is the output of proposed convolutional encoder is modulated with the simplest type of modulation technique used with wireless communication using BPSK modulator. It uses couple phases which are separated by $180^{\circ}$. The output waveform of BPSK is transmitted through AWGN channel. This channel adds white noise to the transmitted data, there is no amplitude loss and phase distortion of frequency components (fading does not exist) to the modulated signals that pass over it. The only distortion is introduced by the noise. If the transmitted signal is denoted as $s(t)$, then the received signal will be [10]:

$$
r(t)=s(t)+n(t)
$$

And the instantaneous channel state information $\mathrm{L}_{\mathrm{c}}$ can be obtained by:

$$
L_{c}=4 a \cdot \frac{E_{b}}{N_{o}}
$$

Where $a$ is fading factor. 
From equation 2, the instantaneous bit error probability of the received bit can be formulated as [10]:

$$
p_{e}(m)=\frac{1}{1+\exp \left[L_{c} \cdot \breve{y}(m)\right]}
$$

Equation (3) shows that, any received data $\breve{y}(\mathrm{~m})$, even $\mathrm{L}_{\mathrm{c}}$ is constant, can be specialized bit error rate. So, $\mathrm{p}_{\mathrm{e}}$ is called instantaneous bit error rate, whilst its mean value equals BPSK bit error rate.

\section{RECEIVER}

The first step at the receiver is to obtain the hard bit and its probability of error, the hard bit and its error probability together called soft bit, the hard bit sequence are moved to the trellis for hard decoding with Viterbi decoder. With MATLAB the poly2trellis is built-in function used for generating all the states and the leaving output of the state. Matrix of information were obtained by flipping; this matrix contains all entering of a particular node in the proposed trellis. With Viterbi algorithm, which considered the best for compute next and previous state, table (1) defines all the transitions of previous state to the next state and the corresponding output for each input bits [9].

The received vector is classified as zero or one using hard and soft decision decoder. Hard decision decoder uses Hamming distance to calculate the branch metrics while soft decision decoder utilizes Euclidian distance to compute the branch metric. By this way, it can be calculating the deviation from corresponding branch bit and get Root of- sum-of-square from each received bit [5].

Next to the convolutional decoder, the output sequence of received bits are moved to the next stage of decoding which is soft-bit decoding, it means the decoder depends on the received hard bit $\mathrm{y}_{\mathrm{o}}(\mathrm{b})$ and its instantaneous error probability $\mathrm{p}_{\mathrm{eo}}(\mathrm{b})$. The conditional bit probabilities can be computed by [4]:

$$
p\left(\frac{y_{o}(b)}{x_{o}^{i}(b)}\right)=\left\{\begin{array}{cl}
1-p_{e o}(b) & \text { if } y_{o}(b)=x_{o}^{i}(b) \\
p_{e o}(b) & \text { if } y_{o}(b) \neq x_{o}^{i}(b)
\end{array}\right.
$$

Conditional probability means that the probability of the transition of a transmitted bit $x_{o}^{i}(b)$ to the known received bit $\mathrm{y}_{\mathrm{o}}(\mathrm{b})$, [7].

Equivalent channel can be considering memoryless channel that leads to find the transition probability for code word as:

$$
p\left(y_{0} / x_{0}^{i}\right)=\prod_{b=1}^{B} p\left(y_{0}(b) / x_{0}^{i}(b)\right)
$$

The self-probability and transition probability are combined to find a posteriori probabilities of transmitted symbol as:

$$
p\left(x_{0}^{i} / y_{0}\right)=\frac{p\left(y_{0} / x_{0}^{i}\right) p\left(x_{0}^{i}\right)}{\sum_{i=0}^{2^{B}-1} p\left(y_{0} / x_{0}^{i}\right) p\left(x_{0}^{i}\right)}
$$

It can be minimized the probability of error, if the error is appeared in the decoded symbol using MAP. MAP is the Maximum of posteriori probability, and it is used to estimate the transmitted encoded symbol as in equation (7). Decoded symbols in MAP represent single codeword from the quantization table [7], [8].

$$
\hat{v}=\max _{i} p\left(x_{0}^{i} / y_{0}\right)
$$




\section{RESULTS AND DISCUSSION}

The performance of the proposed system was evaluated in term of Bit Error Rate (BER), which is common and best term for evaluation of the digital communication system. The simulation is carried out with MATLAB software, and the result with figure (4) shows the BER as a function with energy of bit to noise ratio $\left(E_{b} / N_{0}\right)$. As it's expected the performance of the proposed system with convolutional decoding is better than uncoded BPSK modulation.

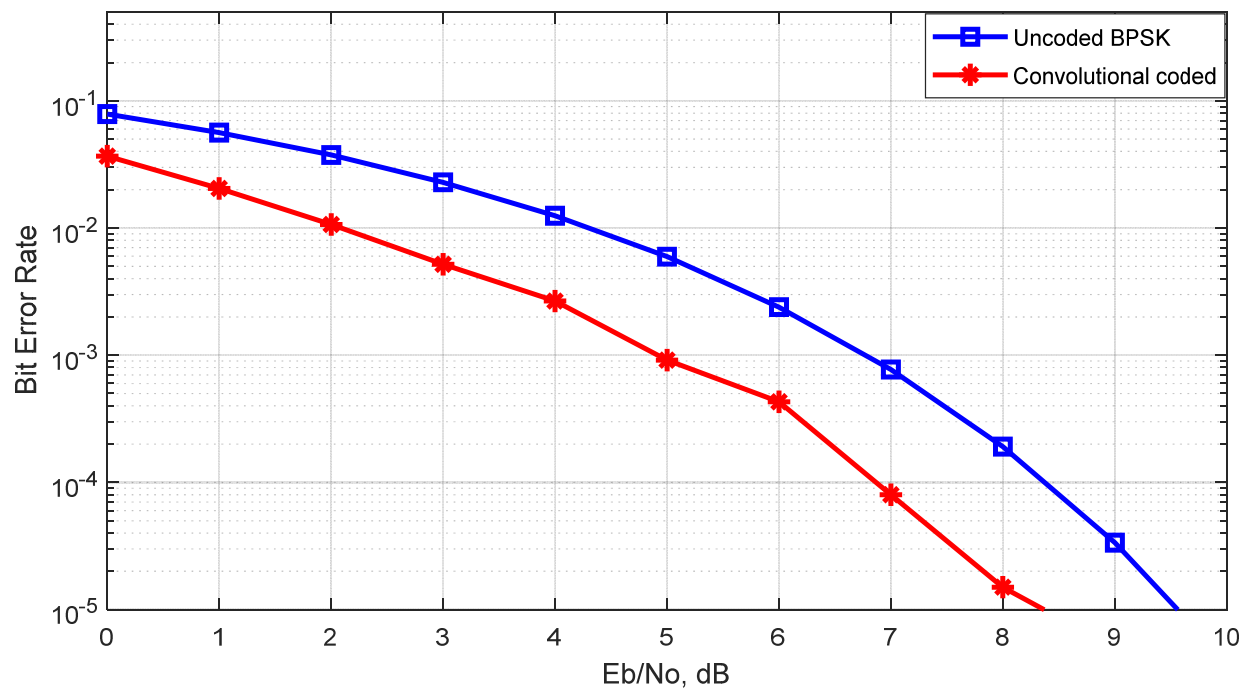

Fig. 4. Convolutional Coded And UnCoded BpsK

While Figure (5) shows the result of simulation of the proposed system which is convolutional decoded aided by soft decoding, this result show the significant improvement obtained in term of BER, this improvement depends on the amount of the priori knowledge.

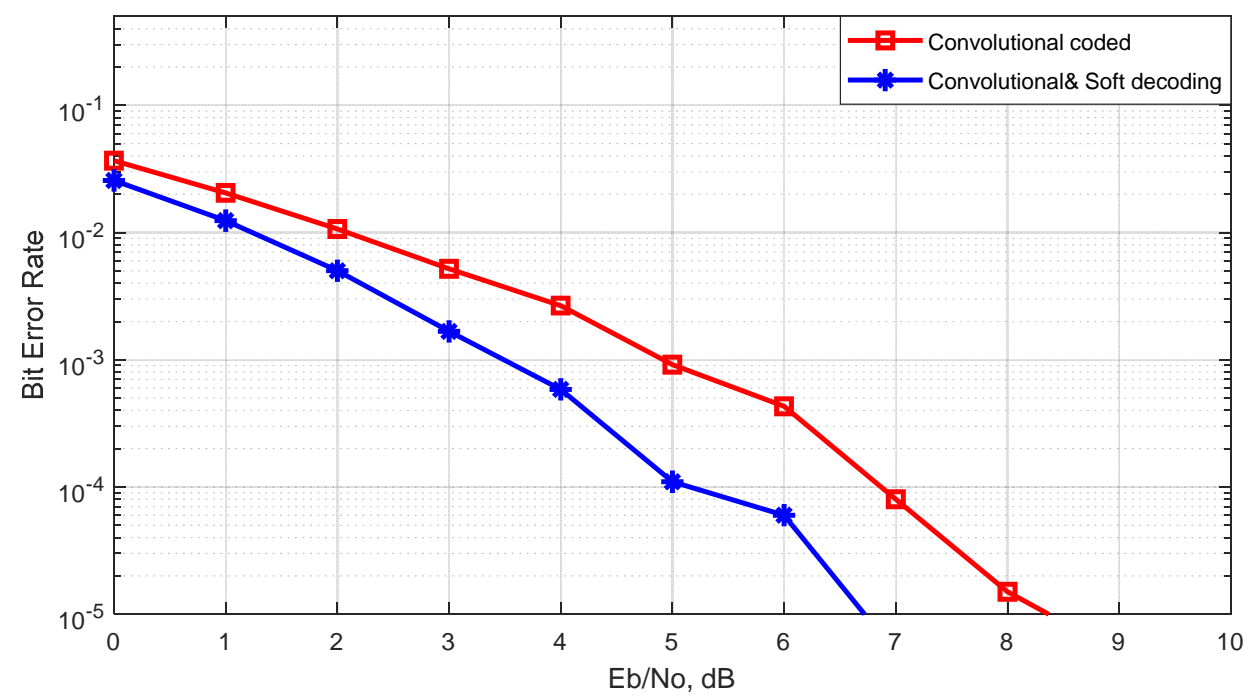

Fig. 5. Convolutional Coded Bpsk Aided By Soft-Bit Decoding

\section{CONCLUSION}

The proposed digital communication system based on convolutional coding as a technique of error control coding has been implemented using MATLAB software, aided by 
soft bit decoding. Performance comparison was carried out between the uncoded BPSK and convolutional coded and the technique of error control coding improves the BER performance. More and significant improvement was obtained when the system aided by soft-bit decoding, the results show that in term of BER this improvement depends on the amount of the priori knowledge.

\section{REFERENCES}

[1] S. Harikumar and M. S, "Performance Evaluation of Convolutional Codes : A MATLAB implementation," ERROR CONTROL CODING PROJECT, pp. 1-4, 2014.

[2] K. Katta, "Design of Convolutional Encoder and Viterbi Decoder using MATLAB," INTERNATIONAL JOURNAL FOR RESEARCH IN EMERGING SCIENCE AND TECHNOLOGY, vol. 1, no. 7, pp. 10-15, 2014.

[3] R. A. Zbaid and K. K. Abdalla, "Design and Implementation of Convolutional Encoder and Viterbi Decoder Using FPGA," Journal of Babylon University/Pure and Applied Sciences, vol. 26, no. 3, 2018.

[4] A. M. Ahmed, "BER Improvement Using Error Concealment Technique for Pulse Code Modulation System," Diyala Journal of Engineering Sciences, 2017.

[5] S. Han and T. Fingscheidt, "VARIABLE-LENGTH VERSUS FIXED-LENGTH CODING:ON TRADEOFFS FOR SOFT-DECISION DECODING," in Acoustics, Speech and Signal Processing (ICASSP), 2014 IEEE International Conference on, Florence, Italy, 2014.

[6] Y. Altug, H. V. Poor and S. Verdti, "On fixed-length channel coding with feedback in the moderate deviations regime," IEEE, pp. 1816-1820, 2015.

[7] T. Fingscheidt and P. Vary, "ROBUST SPEECH DECODING: A UNIVERSAL APPROACH TO BIT ERROR CONCEALMENT," IEEE, pp. 1667-1670, 1997.

[8] S. LIN and D. J. COSTELLO, Error Control Coding fundamental and applications, NEW JERSEY- USA: Prentice- Hall, Inc, Englewood Cliffs, 1983.

[9] J. C. Moreira and P. G. Farrell, ESSENTIALS OF ERROR-CONTROL CODING, The Atrium, Southern Gate, Chichester, UK: John Wiley \& Sons Ltd, 2006.

[10] M. R. Din, Z. H. Peri and D. B. Deni, "Designing Quantizers for Coding Signals with Fixed and Variable Codeword Length," in 23rd Telecommunications forum TELFOR, IEEE, Serbia, Belgrade, 2015. 